\title{
Number, distribution, and predicted needed number of general practitioners in China*
}

\author{
Baoling $\mathrm{Wu}^{1}$, Hanxiang Gong ${ }^{1}$, Zhenni Luo $^{1}$
}

\section{Abstract}

Objective: We aimed to investigate the number and distribution of general practitioners (GPs) in China, and to predict the number of GPs needed in the future.

Methods: From China Health Statistics Yearbook 2014, China Health Statistics Yearbook 2016 and China Statistical Yearbook 2016, we obtained the number of GPs and the populations in China and each of its regions from 2012 to 2015. IBM SPSS Statistics version 22.0 was used to conduct descriptive statistical analysis. Grey model $\operatorname{GM}(1,1)$ and the manpower-to-population ratio method were used to predict the number of GPs needed in China from 2016 to 2020.

Results: The total number of GPs in China was 109,794 in 2012, 145,511 in 2013, 172,597 in 2014, and 188,649 in 2015. The number increased by 78,855 from 2012 to 2015, and the average annual growth rate was $19.77 \%$. By the end of 2015, of the GPs in all of China and in eastern, central, and western China, those who had achieved a GP qualification certificate following training accounted for $63.76 \%(120,285 / 188,649), 60.06 \%(62,474 / 104,015), 65.20 \%(29,565 / 45,344)$, and $71.89 \%(28,246 / 39,290)$, respectively, of the total. The number of GPs per 10,000 population in all of China and in eastern, central, and western China was 1.37, 1.83, 1.05, and 1.06, respectively, and the number of GPs needed for all of China and the eastern, central, and western parts would be $86,275,9787,40,764$, and 34,976 , respectively, calculated on the basis of the standard of two GPs per 10,000 population. Grey model $\operatorname{GM}(1,1)$ predicted that, in accordance with the natural growth trend of GPs, the number of GPs in China would be 220,314 in 2016, 253,728 in 2017, 293,101 in 2018, 339,928 in 2019, and 396,228 in 2020. Moreover, the number of GPs in China in 2020 would be $277,000-415,500$ if the calculated number of GPs to the population ratio is based on the standard of two to three GPs per 10,000 population.

Conclusion: In recent years, although the number of GPs has increased rapidly, GPs are still greatly needed in all of China and in eastern, central, and western China. Moreover, the distribution of GPs is not balanced, and the number of qualified GPs is far from enough. Therefore, team building for GPs should be strengthened, and the training system for GPs should be improved.

Keywords: General practitioners; number; distribution; forecast
1. School of Health Services Management, Guangzhou Medical University, Guangzhou 511436, China

*This article was first published in Chinese in the journal of Chinese General Practice. DOI: 10.3969/j. issn.1007-9572.2018.01.004 CORRESPONDING AUTHOR: Zhenni Luo, Lecturer School of Health Services Management, Guangzhou Medical University, Guangzhou 511436, China

E-mail: janerowe@163.com

Received 13 May 2018; Accepted 20 July 2018

\section{Introduction}

General practitioners (GPs), also called "gatekeepers" of the people, are a new type of physician in China who have received general medicine training. Community health care institutions are their main sites of practice so that they can provide preventive health care for individuals, families, and society, 
diagnosis of, treatment of, and referral for common and frequently occurring diseases, patient rehabilitation, chronic disease management, and health management [1-3]. Since the introduction of the GP system in the late 1980s, the Chinese government and its relevant departments have increasingly attached importance to the development of general medicine. The State Council issued Guiding Opinions on Establishing a General Practitioner System in 2011 [4] to establish a comprehensive GP system and propose the development goal: by 2020, there will be two to three qualified GPs for every 10,000 residents in urban and rural areas. The overall improvement of the GP service will fundamentally meet people's basic medical and health service needs. In recent years, with a growing aging population, changes in the spectrum of diseases, and greater burden of chronic noncommunicable diseases, the role of general medicine and GPs has become increasingly prominent. However, because of the late start of general medicine in China, the short implementation time of the GP system, and the relatively small number of GPs with basic skills, it is urgent to expand the GP team and continuously improve the GP system in the reform of the medical and health system. On this basis, we reviewed data from statistical yearbooks from recent years to analyze the number and distribution of GPs in China, predict their future demand, and explore the development principles to provide the basis for building a GP team and improving the GP system.

\section{Materials and methods}

\section{Data sources}

The number of GPs in this study from 2012 to 2015 and the population data in each region in 2015 were derived from China Health Statistics Yearbook 2014 [5], China Health Statistics Yearbook 2016 [6], and China Statistical Yearbook 2016 [7].

\section{Research methods}

Average annual growth rate: The average annual growth rate was used to reflect the average growth rate and development trend [8]:

Average annual growth rate $=\sqrt[n]{(\text { Final data } / \text { Initial data })}-1$, where $n$ is the number of years minus 1 .
Grey forecasting model GM(1, 1): A grey model (GM) is a forecast model proposed by Deng Julong in the early 1980s. It has been widely used in many fields, such as agriculture and social economy, and has achieved remarkable results. $\operatorname{GM}(1,1)$ is the most basic grey forecasting model in grey theory, with relatively simple calculation and good prediction accuracy. It predicts the future values of a time series, and there is no strict requirement on the sample size and probability distribution. There are some basic steps for building a model of the GM(1, 1) type: (1) accumulate original data sequences; (2) construct the accumulated matrix and constant vector; (3) solve grey parameters; (4) solve $\operatorname{GM}(1,1)$; (5) check the accuracy of the model; (6) make predictions by the mode [9]. Data processing becomes more difficult as it requires complex matrix operations when modeling, which is prone to errors. We used the GM module in the DPS data processing system to analyze the total number of GPs in China from 2012 to 2015. We then established the prediction equations and calculated the error of the predicted value (relative error $=[$ exact value - predicted value]/exact value) so as to predict the total number of GPs in China from 2016 to 2020.

Manpower-to-population ratio approach: The manpower-to-population ratio approach was used to calculate the number of GPs in the target year on the basis of the projected population and allocation criterion of GPs (number of GPs/ population ratio) for that year. The number of GPs in the target year is equal to the population in the target year times the number of GPs divided by the population ratio [10].

Division of economic zones: China was divided into eastern, central, and western regions on the basis of geographic location and economic development. According to the division method of the National Bureau of Statistics and the economic development level, there were 11 subregions in the eastern region (Beijing, Tianjin, Hebei, Liaoning, Shanghai, Jiangsu, Zhejiang, Fujian, Shandong, Guangdong, and Hainan), eight subregions in the central region (Heilongjiang, Jilin, Shanxi, Anhui, Jiangxi, Henan, Hubei, and Hunan), and 12 subregions in the western region (Inner Mongolia, Guangxi, Chongqing, Sichuan, Guizhou, Yunnan, Tibet, Shaanxi, Gansu, Qinghai, Ningxia, and Xinjiang) [11]. 


\section{Statistical methods}

IBM SPSS Statistics version 22.0 was used to conduct descriptive statistical analysis on the number of GPs in China. $\operatorname{GM}(1,1)$ and the manpower-to-population ratio approach were used to predict the future need of GPs in China.

\section{Results}

\section{Number and distribution of GPS in China}

The number of GPs in China was 109,794 in 2012, 145,511 in 2013, 172,597 in 2014, and 188,649 in 2015, with an increase of 78,855 from 2012 to 2015 and an average annual growth rate of $19.77 \%$. There were 1.37 GPs per 10,000 population by the end of 2015, and there was a lack of 86,275 GPs according to the allocation criterion of two GPs per 10,000 population. According to the division of GP institutions, the proportion of GPs in hospitals, community health services, township health centers, and other institutions was $16.64 \%, 38.85 \%, 42.92 \%$, and $1.59 \%$, respectively, as of the end of 2015 .

From 2012 to 2015, the number of GPs in hospitals increased by 10,308 (with an average annual growth rate of $14.19 \%$ ), the number of GPs in community health services increased by 25,425 (with an average annual growth rate of $15.25 \%$ ), the number of GPs in township health centers increased by 42,418 (with an average annual growth rate of $28.06 \%$ ), and the number of GPs in other institutions increased by 704 (with an average annual growth rate of $9.31 \%$ ); see Table 1 .

\section{Distribution of GPs in different regions of China in 2015}

As of the end of 2015, the proportions of GPs with a GP training certificate in all of China and in the eastern, central, and western regions of China were $63.76 \%(120,285 / 188,649)$,
$60.06 \% \quad(62,474 / 104,015), \quad 65.20 \% \quad(29,565 / 45,344)$, and $71.89 \%$ (28,246/39,290), respectively. Yunnan (81.93\%), Sichuan $(79.13 \%)$, and Hebei $(76.76 \%)$ had the highest proportion of GPs with GP certificates, and Tibet (29.19\%), Shanghai (31.03\%), and Beijing (47.47\%) had the lowest.

The number of GPs per 10,000 population in all of China and in the eastern, central, and western regions of China was $1.37,1.83,1.05$, and 1.06, respectively. In 2015, Zhejiang (3.90) had the highest ratio of GPs per 10,000 population, followed by Beijing (3.81) and Shanghai (3.04). Tibet (0.50), Shaanxi (0.56), and Jiangxi (0.73) had the lowest ratio. The shortage of GPs in all of China and in the eastern, central, and western regions of China was 86,275, 9,787, 40,764, and 34,976, respectively, according to the allocation criterion of two GPs per 10,000 population (see Table 2).

\section{$G M(1,1)$ prediction results}

Model fitting of GPs in China from 2013 to 2015 was done in $\operatorname{GM}(1,1)$ of the DPS grey system module, and the fitting effect was good $(C=0.0118, P=1.0000)$. The exact number of GPs in China was 145,511 in 2013, 172,597 in 2014, and 188,649 in 2015. The predictions were 145,880 in $2013,172,146$ in 2014 , and 188,837 in 2015 , and the relative errors were $-0.25 \%$, $0.26 \%$, and $-0.10 \%$, respectively. $\operatorname{GM}(1,1)$ predicted that the total number of GPs in China would be 220,314 in 2016, 253,728 in 2017, 293,101 in 2018, 339,928 in 2019, and 396,228 in 2020 according to the annual growth rate (see Fig. 1).

\section{Forecast results of the manpower-to-population ratio approach}

The forecast results for the future population of China showed that China's population would reach at least 1.385 billion by

Table 1. Number and percentage of general practitioners in different institutions in China from 2012 to 2015

\begin{tabular}{llllll}
\hline Year & Total & Hospitals & Community health services & Township health centers & Other institutions \\
\hline 2012 & 109,794 & $21,074(19.19 \%)$ & $47,863(43.59 \%)$ & $38,557(35.12 \%)$ & $2300(2.10 \%)$ \\
2013 & 145,511 & $25,758(17.70 \%)$ & $60,181(41.36 \%)$ & $56,825(39.05 \%)$ & $2747(1.89 \%)$ \\
2014 & 172,597 & $30,428(17.63 \%)$ & $68,914(39.93 \%)$ & $70,296(40.73 \%)$ & $2959(1.71 \%)$ \\
2015 & 188,649 & $31,382(16.64 \%)$ & $73,288(38.85 \%)$ & $80,975(42.92 \%)$ & $3004(1.59 \%)$ \\
Increase in number & 78,855 & 10,308 & 25,425 & 42,418 & 704 \\
Average annual growth rate (\%) & 19.77 & 14.19 & 15.25 & 28.06 & 9.31 \\
\hline
\end{tabular}


Table 2. Data on general practitioners (GPs) in China in 2015

\begin{tabular}{|c|c|c|c|c|c|}
\hline Region & $\begin{array}{l}\text { Total } \\
\text { number } \\
\text { of GPs }\end{array}$ & $\begin{array}{l}\text { Number registered } \\
\text { in a general } \\
\text { medicine specialty }\end{array}$ & $\begin{array}{l}\text { Number with a GP } \\
\text { training certificate }\end{array}$ & $\begin{array}{l}\text { Number of GPs } \\
\text { per } 10,000 \\
\text { population }\end{array}$ & $\begin{array}{l}\text { Shortage of GPs under the criterion } \\
\text { of two general practitioners per } \\
10,000 \text { people population }\end{array}$ \\
\hline All China & 188,649 & $68,364(36.24 \%)$ & $120,285(63.76 \%)$ & 1.37 & 86,275 \\
\hline Eastern & 104,015 & $41,541(39.94 \%)$ & $62,474(60.06 \%)$ & 1.83 & 9787 \\
\hline Central & 45,344 & $15,779(34.80 \%)$ & $29,565(65.20 \%)$ & 1.05 & 40,764 \\
\hline Western & 39,290 & $11,044(28.11 \%)$ & $28,246(71.89 \%)$ & 1.06 & 34,976 \\
\hline Beijing & 8269 & $4344(52.53 \%)$ & $3925(47.47 \%)$ & 3.81 & -3927 \\
\hline Tianjin & 2144 & $661(30.83 \%)$ & $1483(69.17 \%)$ & 1.39 & 950 \\
\hline Hebei & 9286 & $2158(23.24 \%)$ & $7128(76.76 \%)$ & 1.25 & 5564 \\
\hline Shanxi & 4014 & $1460(36.37 \%)$ & $2554(63.63 \%)$ & 1.10 & 3314 \\
\hline Inner Mongolia & 3085 & $1084(35.14 \%)$ & $2001(64.86 \%)$ & 1.23 & 1937 \\
\hline Liaoning & 3624 & $1039(28.67 \%)$ & $2585(71.33 \%)$ & 0.83 & 5140 \\
\hline Jilin & 2.891 & $933(32.27 \%)$ & $1958(67.73 \%)$ & 1.05 & 2615 \\
\hline Heilongjiang & 4320 & $1266(29.31 \%)$ & $3054(70.69 \%)$ & 1.13 & 3304 \\
\hline Shanghai & 7352 & $5071(68.97 \%)$ & $2281(31.03 \%)$ & 3.04 & -2522 \\
\hline Jiangsu & 20,841 & $7986(38.32 \%)$ & $12,855(61.68 \%)$ & 2.61 & -4889 \\
\hline Zhejiang & 21,627 & $8134(37.61 \%)$ & $13,493(62.39 \%)$ & 3.90 & $-10,549$ \\
\hline Anhui & 7360 & $2955(40.15 \%)$ & $4405(59.85 \%)$ & 1.20 & 4928 \\
\hline Fujian & 5122 & $1470(28.70 \%)$ & $3652(71.30 \%)$ & 1.33 & 2556 \\
\hline Jiangxi & 3319 & $1147(34.56 \%)$ & $2172(65.44 \%)$ & 0.73 & 5813 \\
\hline Shandong & 9920 & $2836(28.59 \%)$ & $7084(71.41 \%)$ & 1.01 & 9774 \\
\hline Henan & 10,349 & $3247(31.38 \%)$ & $7102(68.62 \%)$ & 1.09 & 8611 \\
\hline Hubei & 6970 & $2254(32.34 \%)$ & $4716(67.66 \%)$ & 1.19 & 4734 \\
\hline Hunan & 6121 & $2517(41.12 \%)$ & $3604(58.88 \%)$ & 0.90 & 7445 \\
\hline Guangdong & 14,955 & $7439(49.74 \%)$ & $7516(50.26 \%)$ & 1.38 & 6743 \\
\hline Guangxi & 4671 & $1339(28.67 \%)$ & $3332(71.33 \%)$ & 0.97 & 4921 \\
\hline Hainan & 875 & $403(46.05 \%)$ & $472(53.94 \%)$ & 0.96 & 947 \\
\hline Chongqing & 2872 & $773(26.92 \%)$ & $2099(73.08 \%)$ & 0.95 & 3162 \\
\hline Sichuan & 10,394 & $2169(20.87 \%)$ & $8225(79.13 \%)$ & 1.27 & 6014 \\
\hline Guizhou & 3147 & $1403(44.58 \%)$ & $1744(55.42 \%)$ & 0.89 & 3913 \\
\hline Yunnan & 4289 & $775(18.07 \%)$ & $3514(81.93 \%)$ & 0.90 & 5195 \\
\hline Tibet & 161 & $114(70.81 \%)$ & $47(29.19 \%)$ & 0.50 & 487 \\
\hline Shaanxi & 2126 & $599(28.17 \%)$ & $1527(71.83 \%)$ & 0.56 & 5460 \\
\hline Gansu & 3312 & $1064(32.13 \%)$ & $2248(67.87 \%)$ & 1.27 & 1888 \\
\hline Qinghai & 961 & $263(27.37 \%)$ & $698(72.63 \%)$ & 1.63 & 215 \\
\hline Ningxia & 565 & $196(34.69 \%)$ & $369(65.31 \%)$ & 0.85 & 771 \\
\hline Xinjiang & 3707 & $1265(31.12 \%)$ & $2442(65.88 \%)$ & 1.57 & 1013 \\
\hline
\end{tabular}

The number of GPs refers to the total number of practicing (assistant) physicians who are registered in a GP specialty or who have obtained GP training certificates. Since the number of people in each region in China Statistical Yearbook 2016 is rounded to a single digit and in the tens of thousands, the total number may be biased, which means the shortage of GPs is not equal to the sum of GPs in all regions under the allocation criterion of two GPs per 10,000 population. 


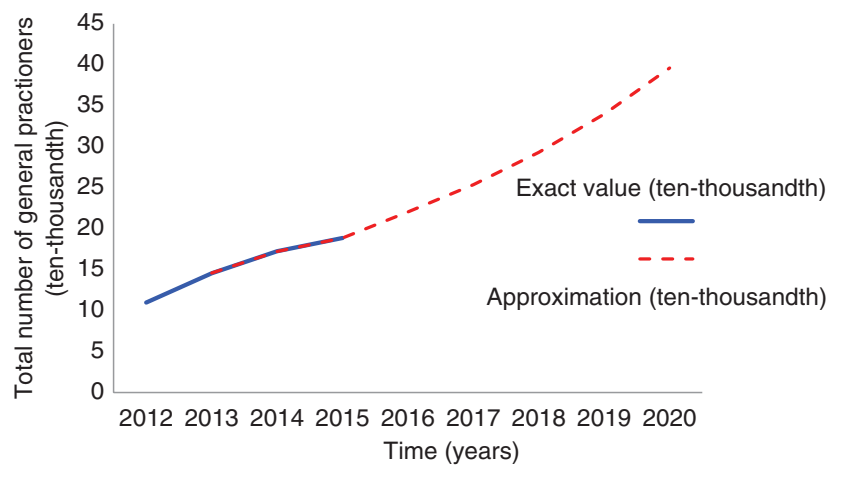

Fig. 1. Exact number of general practitioners in China from 2013 to 2015 and number of general practitioners in China from 2013 to 2020 predicted with grey model GM(1, 1).

2020 [12]. In December 2016, the State Council issued the 13th Five-Year Plan for Healthcare (state issue [2016] no. 7) and proposed the target with binding effect: by 2020, there should be at least two GPs for every 10,000 population in China. According to this minimum allocation standard, the manpower-to-population ratio approach calculated that the number of GPs in China would be at least 277,000 in 2020. With the allocation standard of three GPs per 10,000 population, the number of GPs in China would be 415,500 in 2020.

\section{Discussion and recommendations}

\section{Uneven distribution of general practitioners in China}

This study showed that the number of GPs in China increased by 78,855 from 2012 to 2015, with an average annual growth rate of $19.77 \%$. With regard to different institutions, the number of GPs in township health centers grew the fastest compared hospitals and community health services, with an average annual growth rate of $28.06 \%$. This resulted from the establishment of a comprehensive GP system in 2011 and the government's input and investment. It also showed that Zhejiang, Beijing, and Shanghai had more than three GPs for every 10,000 population, while Tibet, Shaanxi, and Jiangxi had less than one in 2015. The uneven distribution of the number of GPs may be closely associated with the different economic development level in different regions, suggesting that attention should be paid to the fairness and accessibility of health services in various regions, especially in remote areas where the economy is lagging behind, when GP resources are allocated.

\section{Strengthen the general practitioner team}

The $\operatorname{GM}(1,1)$ prediction results showed that the total number of GPs in China would be 396,228 under the assumption of the annual growth rate in 2020. However, according to the allocation criteria of two or three GPs per 10,000 population, the number of GPs in China should be 277,000-415,500 in 2020, as calculated by the manpower-to-population ratio approach. Therefore, another 88,351-226,851 GPs need to be trained on the basis of the number of GPs in 2015. From the number of GPs per 10,000 population in each region, only Zhejiang, Beijing, Shanghai, and Jiangsu have more than two GPs for every 10,000 population. This shows that the shortage of GPs is generally widespread, whether on a national level or on a regional level, far from meeting the increasing health needs of the Chinese people. With the increasing pressure on GPs, the state must expand the GP team. First, the government should focus on the top-level design of the GP team and promote the development of this team through policy and market guidance. Second, GPs usually do not want to "stay" and "serve in community service centers." This problem should be solved by improving the legal security system and incentive mechanisms for GPs to attract more people to the team [13].

\section{Improve the quality of general practitioners while increasing their number}

This study indicated that the number of GPs in China will continue to grow. As of the end of 2015, the number of GPs who had obtained GP training certificates was 120,285, accounting for $63.72 \%$ of the total number of GPs, but the proportion in some areas was less than $50.0 \%$. However, as of the end of 2015, China had an average of 0.87 qualified GPs per 10,000 population, and there is still a huge gap between the target of two to three qualified GPs per 10,000 population. Therefore, while the number is increasing, it is a top priority to produce more qualified GPs to truly play the role of "health gatekeepers" for the Chinese people. With the rapid growth of GPs at the grassroots level, the faculty team of GPs should be improved to ensure the quality of GPs. A core team of GPs with strong capabilities and a high level of teaching can support the sustainable development of the GP training system and promote the coordinated development of performing, teaching, and research among GPs [14]. Therefore, teaching 
training programs for key GPs can be conducted nationwide by hiring domestic and foreign outstanding general medicine experts and encouraging medical institutions at all levels to support continuing education of GPs.

As time is limited and the tasks are demanding, China should improve the system and efficiency of GP training to produce more GPs. The current modes of GP training in China mainly are the " $5+3$ " mode, the " $3+2$ " mode, and transfer training. The " $5+3$ " mode is used to integrate the training of GPs and the training of graduate students in general medicine. It is the mainstream mode, or the only target mode to train Chinese GPs [15]. The stages in the " $5+3$ " mode mainly include a rotation stage in clinical departments, a community practice stage, and a related theory training stage. Community practice is the practical stage for GPs, and should focus on the application of the basic theory of general medicine, knowledge of clinical diseases, skills of chronic disease management, and knowledge of behavioral science, humanities, sociology, and other disciplines related to the daily work of GPs [15].

\section{Conflict of interest}

The authors declare that they have no conflict of interest.

\section{Funding}

National Natural Science Foundation of China (Grant No. 71503057).

\section{Author contributions}

Wu Baoling conducted experimental design and implementation, paper writing, and completion, and is responsible for the article. Gong Hanxiang conducted experimental implementation, evaluation, and data collection; Luo Zhenni conducted quality control and proofreading.

\section{Significance Statement}

Analysis of the number and distribution of general practitioners in China from 2012 to 2015 based on China Health Statistics Yearbook 2014, China Health Statistics Yearbook 2016 and China Statistical Yearbook 2016. Grey model and manpowerto-population ratio approach to predict future demand of general practitioners in China from 2016 to 2020.

\section{References}

1. Li CF, Yin AT, Wang GW, et al. Studying on the construction of general practitioner system based on connotation construction. Chin Health Serv Manag 2015;32(7):512-4.

2. Dai T, Ju H, Ma XJ. Development of international general practice system: influencing factors and policy implications. Chin J Health Policy 2015;8(2):1-7.

3. Lin DH. Status and countermeasures of standardized training of general practitioners in community in Fujian province. Fuzhou: Fujian Medical University; 2014.

4. State Council Guiding opinions on establishing a general practitioner system; 2011.

5. National Health and Family Planning Commission. China health statistics yearbook 2014. Beijing: Peking Union Medical College Press; 2014.

6. National Health and Family Planning Commission. China health statistics yearbook 2016. Beijing: Peking Union Medical College Press; 2016.

7. National Bureau of Statistics of the People's Republic of China. China statistical yearbook 2016. Beijing: China Statistics Press; 2016.

8. Bai J, Lu Y, Yang FH, et al. Changes of community public health services in Chaoyang District of Beijing between 2010 and 2012 Chin Gen Pract 2015;18(13):1514-7.

9. Deng JL. The primary methods of grey system theory. Wuhan: Huazhong University of Science and Technology Press; 1987.

10. Shen X, Lu F. Demand analysis and prediction research of rural doctors by human population ratio method. Chin Rural Health Serv Admin 2016;36(3):289-92.

11. Wang ZP, Zhai JT, Zheng D. Comparative study on the performance of urban land reserve decision among eastern, central and western areas of China. Areal Res Dev 2016;35(4):120-5.

12. Sun MZ. A forecast of Chinese future population through the data of sixth census - the population decay of future China. Soc Sci Beijing 2014;29(5):85-92.

13. Li SJ, Zhang HR, Zhu LN, et al. Status quo of occupational pressure and job satisfaction of general practitioners and its influencing factor. Chin Gen Pract 2015;18(4):387-90.

14. Fang CM, Zhang P, Shen TB, et al. Approach and effect of the general practice teacher training in Zhejiang province. Chin Gen Pract 2015;18(7):807-10.

15. Chen SL, Hu YY, Wang B, et al. Mastery status of and training demands for general practice knowledge and skills of general practitioners in Hubei province. Chin Gen Pract 2015;18(13):1555-9. 